\title{
Welche Patientin mit frühem Mammakarzinom braucht eine Chemotherapie?
}

\section{1-Gen-Expressions-Assay hilft bei der Therapieplanung}

\begin{abstract}
Anlässlich der 12th St. Gallen International Breast Cancer Conference hat Genomic Health, Inc. neue Daten zum Einsatz des Oncotype DX ${ }^{\circledR}$ Breast Cancer Assay präsentiert. Insgesamt liegen damit nun die Daten von 11 Decision-Impact-Studien vor, die unisono zeigen, dass die Ergebnisse dieses Tests in etwa einem Drittel aller Fälle von Brustkrebs im Frühstadium die Therapieempfehlungen der behandelnden Ärzte verändern. In vielen Fällen kann so eine Chemotherapie eingespart werden.
\end{abstract}

Beim Oncotype DX ${ }^{\circledR}$-Test wird aus einer Tumorprobe die Expression von 21 Genen, darunter 16 krebsspezifischen und 5 ReferenzGenen, bestimmt. Daraus wird ein «Recurrence Score ${ }^{\circledR}{ }_{\gg}$ mit einem Wert zwischen 0 und 100 Punkten errechnet; je höher er ausfällt, desto größer ist das Rezidivrisiko in den nächsten 10 Jahren. Diese Information hilft dem behandelnden Arzt bei der Entscheidung über die adjuvante Therapieplanung.

In der deutschen Decision-Impact-Studie, so Prof. Dr. Wolfgang Eiermann, ärztlicher Direktor der Frauenklinik am Rotkreuzklinikum in München, waren insgesamt 360 Patientinnen eingeschlossen, darunter auch solche mit nodal positivem Brustkrebs, d.h. mit 1-3 befallenen Lymphknoten. In einer Zwischenauswertung von 197 Studienteilnehmerinnen zeigte sich, dass bei 38\% die Therapieempfehlungen aufgrund des Testergebnisses geändert worden waren; bei $24 \%$ der Patientinnen wurde statt einer kombinierten Chemohormontherapie nur

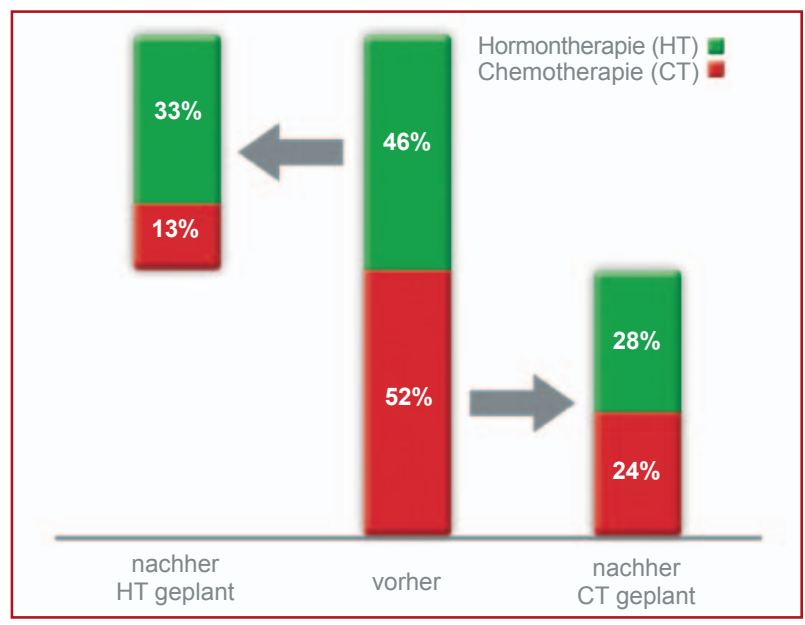

eine endokrine Therapie verabreicht, bei $13 \%$ musste aufgrund des Testergebnisses die Hormontherapie (HT) um eine Chemotherapie (CT) ergänzt werden (Abb. 1). Bei den 48 Patientinnen mit nodal positiven Tumoren wurde sogar in $48 \%$ der Fälle der Therapieplan geändert (bei 35\% ein Verzicht auf Chemotherapie, bei $13 \%$ die zusätzliche Durchführung). Nach Kenntnis des individuellen Recurrence Scores ${ }^{\circledR}$ erhielten insgesamt $63,4 \%$ der Patientinnen letztlich keine Chemotherapie; das entspricht einer Netto-Reduktion um 17\%.

Diese Resultate belegen laut Eiermann, dass der Oncotype DX ${ }^{\circledR}$-Test auch in der klinischen Praxis in Deutschland Auswirkungen auf die Therapieentscheidung beim frühen Mammakarzinom hat und dass dadurch der Einsatz von Chemotherapien insgesamt zurückgefahren werden kann, insbesondere bei Frauen mit nodal positiven Tumoren.

In St. Gallen wurden außerdem die Resultate einer Metaanalyse von 7 Decision-Impact-Studien vorgestellt, an denen insgesamt 912 Patientinnen teilgenommen hatten: Dabei kam es aufgrund der Ergebnisse von Oncotype DX ${ }^{\circledR}$ bei 37\% der Patientinnen zu einer Änderung der ursprünglichen Therapieempfehlung. Bei 33\% der Teilnehmerinnen war das ein Verzicht auf die ursprünglich empfohlene Chemotherapie, be 4\% wurde eine geplante Hormontherapie um eine Chemotherapie ergänzt.

«Der Oncotype DX ${ }^{\circledR}$-Test beeinflusst fundamental unsere therapeutischen Entscheidungen», erklärte Eiermann. «Seine Verfügbarkeit ist ein wichtiger Schritt in Richtung personalisierter Medizin. Es gibt einen eindeutigen Bedarf, dieses diagnostische Verfahren in die täg-

Abb. 1. Die deutsche Decision-Impact-Studie: Die Abbildung veranschaulicht, wie der Oncotype DX ${ }^{\circledR}$ Recurrence Score die Entscheidung für oder gegen eine Chemotherapie beeinflusst. liche klinische Praxis zu integrieren.» Auch Prof. Dr. Jens-Uwe Blohmer, Chefarzt der Abteilung für Frauenheilkunde und Geburtshilfe am Brustzentrum City des Sankt Gertrauden-Krankenhauses Berlin, bestätigte: «Das Ergebnis von Oncotype DX ${ }^{\circledR}$ liefert mir zusätzliche Argumente, sodass ich mich als onkologisch tätiger Arzt in meinen Therapieempfehlungen sicherer fühle.»

Zuversichtlich zeigte sich Eiermann bezüglich der Erstattung der Kosten für den Test durch die Kassen: Eine Kostenersparnis sei mit Sicherheit anzunehmen, die genauen Daten werden derzeit in einer Studie untersucht.

\section{Charakteristika von Oncotype DX ${ }^{\circledR}$}

- Der Oncotype DX ${ }^{\circledR}$-Test wurde in bislang insgesamt 13 klinischen Studien mit weltweit mehr als 4.000 Brustkrebs-Patientinnen intensiv evaluiert $[1,2]$. Bis heute haben rund 10.000 Ärzte in über 60 Ländern Oncotype $\mathrm{DX}^{\circledR}$ be mehr als 190.000 Frauen eingesetzt.

- $\quad$ Ein besonderer Vorteil von Oncotype DX ${ }^{\circledR}$ ist, dass der Assay mit Proben aus vorhandenem in Paraffin eingebettetem Tumorgewebe funktioniert. Es ist also weder ein zusätzlicher Eingriff zur Asservierung von Frischgewebe noch eine besondere Gewebeaufbereitung erforderlich.

- Die Anwendung von Oncotype DX ${ }^{\circledR}$ wird in den Leitlinien der Deutschen Gesellschaft für Hämatologie und Onkologie (DGHO), der American Society of Clinical Oncology (ASCO) und des National Comprehensive Cancer Network (NCCN) empfohlen.

Weitere Informationen

- Kostenfreie Hotline: 00800-66268973

- www.oncotypedx.de

- germansupport@genomichealth.com

Josef Gulden, Grafrath

\section{Quelle}

European Media Conference «Chemo? No Chemo? The newly available Oncotype DX Breast Cancer Assay helps you find an answer» am 16. März 2011 in Zürich, veranstaltet von Genomic Health, Inc.

\section{Referenzen}

1 Paik S et al.: N Engl J Med 2004;351:2817-26.

Dowsett M et al.: J ClinOncol 2010;28:1829-34.

\section{Impressum}

Welche Patientin mit frühem Mammakarzinom braucht eine Chemotherapie?

21-Gen-Expressions-Assay hilft bei der Therapieplanung

Innovationen in BREAST CARE 6 | 2 | 11

(c) 2011 by S. Karger Verlag für Medizin und Naturwissenschaften $\mathrm{GmbH}$

Wilhelmstraße 20A

79098 Freiburg, Deutschland

Mit freundlicher Unterstützung durch Genomic Health, Inc.

Verlag, Herausgeber, Redaktion und Verlagsgeschäftsführung übernehmen keine Verantwortung für den Inhalt dieser Rubrik. 